\title{
Touch Sensitivity of Objects Based on Surface Acoustic Waves
}

\author{
C. Yü ${ }^{1}$, S. Krämer ${ }^{2}$, D.Wieland ${ }^{2}$, S. Krempel ${ }^{2}$ and G. Lindner ${ }^{2}$ \\ ${ }^{1}$ School of Optical-Electrical and Computer Engineering, University of Shanghai for Science and \\ Technology, No. 516, Jungong Rd., Shanghai, P.R. China \\ kennie.yucheng@googlemail.com \\ ${ }^{2}$ Institution of Sensor and Actuator Technology, Coburg University of Applied Sciences and Arts, Am \\ Hofbräuhaus 1, 96450 Coburg, Germany
}

\begin{abstract}
:
With the mode conversion effect of surface acoustic waves, a touch sensitivity of solid plates can be achieved without affecting the front side but by attaching transducers to the backside of the plate. By thinning the region of the surface acoustic wave transmission to a thickness smaller than the wavelength, a large touch sensitivity of about $70 \%$ has been achieved at different materials such as ceramics, metals and plastics. This is accompanied by mode splitting and dispersion, which may provide an access for internal referencing with respect to aging and temperature influences. Prospectic applications of this touch sensing concept are tiles with switching functions in bathrooms or touch control panels at medical equipments, in kitchens or in cars.
\end{abstract}

Key words: Touch sensing, surface acoustic waves, dispersive Lamb waves, mode conversion, functional tiles

\section{Introduction}

Touch sensitivity of solid surfaces can be achieved by the mode conversion of surface acoustic waves propagating on those surfaces at the contact area with human tissue, if the phase velocity of the surface acoustic wave is larger than the velocity of sound in tissue [1, 2]. This concept has been realized at ceramic tiles by attaching two piezoelectric single phase transducers to the backside of the tiles, forming emitter and receiver of a surface acoustic wave transmission line. The pitch of the electrode structure of the transducer, which defines the wavelength of the surface acoustic waves, has been chosen to be in the order of the thickness of the tile. In this case the surface acoustic wave excited on the backside resulted in smaller, but still detectable displacements on the front side and thus a touch sensitivity of the tile there [3].

The touch effects on the receiver signal, however, remained rather small (about 10 to $20 \%$ reduction of signal amplitude) and there was no reference signal available which could be used for the compensation of temperature influence or aging effects. Therefore the construction has been changed by reducing the thickness of the substrate in the region of the acoustic surface wave transmission line by milling from the backside and attaching piezoelectric single phase transducers with unchanged pitch to this thinner area of the substrate. Thus the ratio of substrate thickness to wavelength was reduced compared to the previous version, which changed the type of surface acoustic waves towards Lamb waves, exhibiting a splitting of modes with different propagation velocities and a dispersion of those modes, i.e. a dependence of the phase velocity of the frequency $[4,5]$.

This concept has been realized with substrate plates made of different solid substrates and in all cases an improvement of the touch sensitivity has been achieved. In addition, mode splitting and dispersion phenomena have been observed which may provide an access towards internal referencing with respect to temperature and aging compensation. Results obtained so far with samples of this type are presented in the following.

\section{Sensor construction and measurement concept}

Rectangular plates of different materials ceramic tiles, aluminum, PVC, polypropylene (PP), phenolic resin with cotton fibers - with an area of $100 \times 150 \mathrm{~mm}^{2}$ and a thickness of $6 \mathrm{~mm}$ were used as substrates for the experiments. In the center of the plates a rectangular area of 11 
$x 50 \mathrm{~mm}^{2}$ was thinned down to thicknesses of $\mathrm{d}$ $=0.5-2.5 \mathrm{~mm}$ by milling from the backside (Fig. 1). Near the edges of those thinned regions two piezoelectric single phase transducers made of PZT were attached to the thin part of the substrate at a distance of $50 \mathrm{~mm}$ by gluing and were contacted to a function generator and an oscilloscope for excitation and detection of surface acoustic waves, respectively. The single phase transducers were equipped with a horseshoe-type electrode adjacent to the substrate surface with a pitch of $3 \mathrm{~mm}$ between the fingers of the electrode, thus implying a wavelength of $\lambda=3 \mathrm{~mm}$ to surface acoustic waves generated on the substrates. Surface acoustic waves will be excited at frequencies $f$ for which the relationship is written as Eq. (1).

$$
\mathrm{c}_{\mathrm{ph}}=\lambda \cdot \mathrm{f}
$$

$\mathrm{c}_{\mathrm{ph}}$, the phase velocity of the surface acoustic wave, will be fulfilled. Since in the thinned regions $d<\lambda$ and thus the surface acoustic waves are of the Lamb wave type there, a splitting of modes into symmetrical and antisymmetrical modes with different phase velocities and a dependence of the phase velocities from the frequency will be expected there. Therefore, at least with respect to the fundamental mode, two sub-modes $\left(A_{0}\right.$ and $\left.S_{0}\right)$ will be excited, which are propagating with different velocities. According to the velocityfrequency relationship they will appear at different excitation frequencies and their transmission time along the fixed distance between the two transducers will be different. In addition, according to the dispersive character of Lamb waves, their characteristic frequencies will differ slightly for different substrate thicknesses.

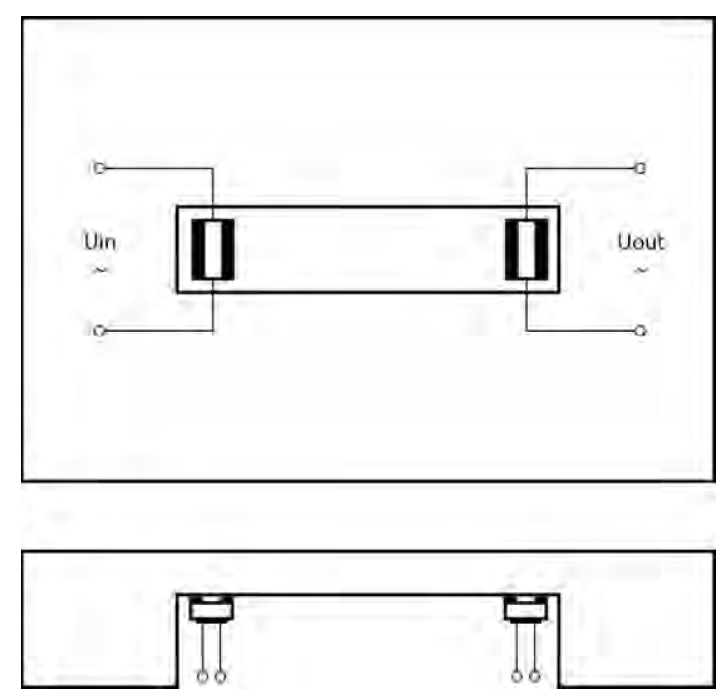

Fig. 1. Schematic drawing of construction of samples. View from backside showing the thinned region and the transducers attached there (above); Cross-section along the sagittal plane across the thinned region (below).

Measurements of the transmission of surface acoustic waves along the thinned part of the substrate were performed by exciting on one the transducers with a sinusoidal alternating voltage from a function generator with variable frequency, whereas the second transducer was connected with a digital storage oscilloscope. In order to achieve a proper response of the transducer to the changing frequencies in the range of 200 to $2000 \mathrm{kHz}$, a burst excitation with five cycles and a peak-to-peak voltage of $20 \mathrm{~V}_{\mathrm{pp}}$ was chosen. Correspondingly, a receiver signal was detected after a transmission time, which is related to the propagation velocity of the acoustic wave and which consists of a sequence of five cycles with subsequently increasing amplitude followed by additional cycles with decreasing amplitude reflecting the transient response of the transducers. The transmission time was determined from the time difference between the switch-off of the excitation pulse and the zero-crossing of the receiver signal following the maximum amplitude.

For the measurement of the touch sensitivity, a mode conversion tester was used, which consisted of a stamp made of silicon in its lower part, which was pressed to the front side of the substrate by a weight of $1 \mathrm{~kg}$ with ultrasonic gel in between. This tester should allow a quantitative comparison between the touch sensitivities of different wave modes and substrates. The touch effect itself, however, was checked in any case with a human finger in addition.

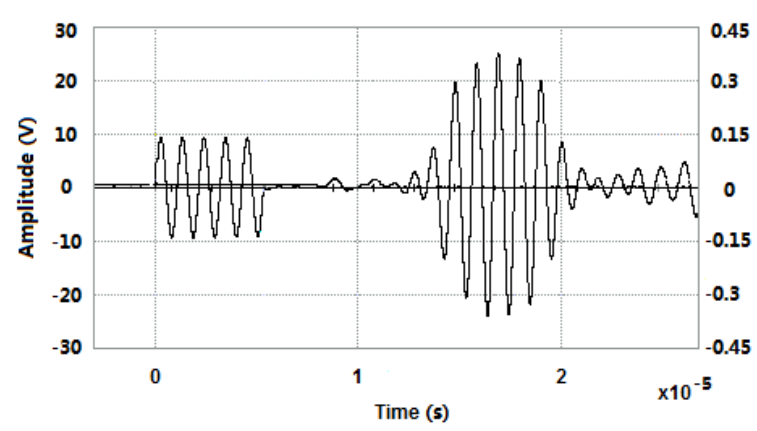

Fig. 2. Sinusoidal burst excitation signal (left) and receiver signal (right) as recorded by a storage oscilloscope at an aluminum substrate (excitation frequency $928 \mathrm{kHz}$, substrate thickness $1 \mathrm{~mm}$ ).

\section{Results}

At all samples tested so far wave modes were observed which exhibited large touch sensitivities, which were restricted to the 
thinned area between the two transducers. For instance, at a PVC substrate thinned to $1 \mathrm{~mm}$, a strong receiver signal was obtained at frequency of $342 \mathrm{kHz}$ with a maximum peak-topeak voltage amplitude of $233 \mathrm{mV}$ and a transit time of $22.6 \mu \mathrm{s}$, which corresponds to a propagation velocity of $2212 \mathrm{~m} / \mathrm{s}$. Applying the mode conversion tester, this signal was reduced to $81 \mathrm{mV}$, i.e. by $65 \%$, whereas the transit time with $22.9 \mu \mathrm{s}$ remains almost unchanged (Fig. 3). Similar observations were recorded with aluminum substrates and with other substrates; they are compiled in table 1.
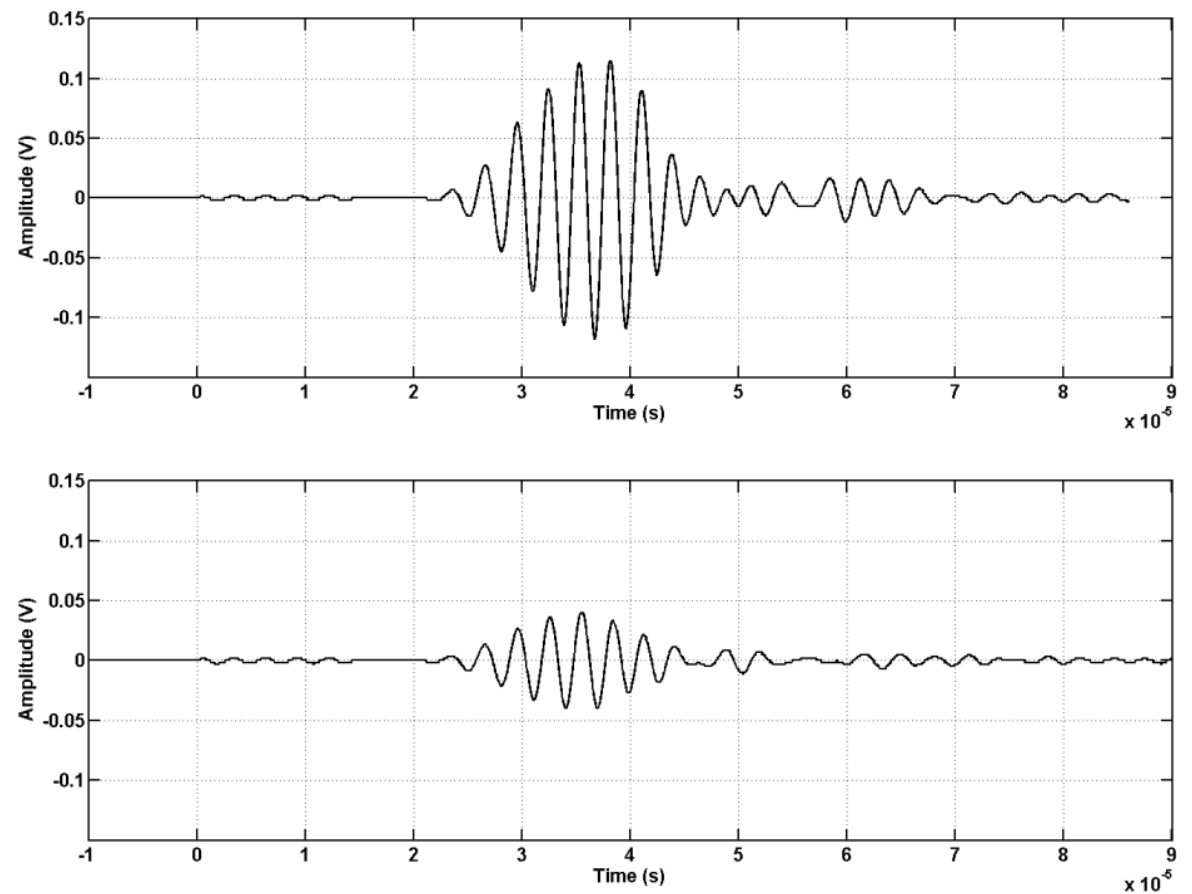

Fig. 3. Receiver signals measured as a function of time after starting burst excitation at a PVC substrate with $1 \mathrm{~mm}$ thickness at an excitation frequency of $342 \mathrm{kHz}$ without (above) and with applying a mode conversion tester (below). The weak signal observable between 0 and $14 \mu$ s transit time are come by capacitive coupling between the two transducers.
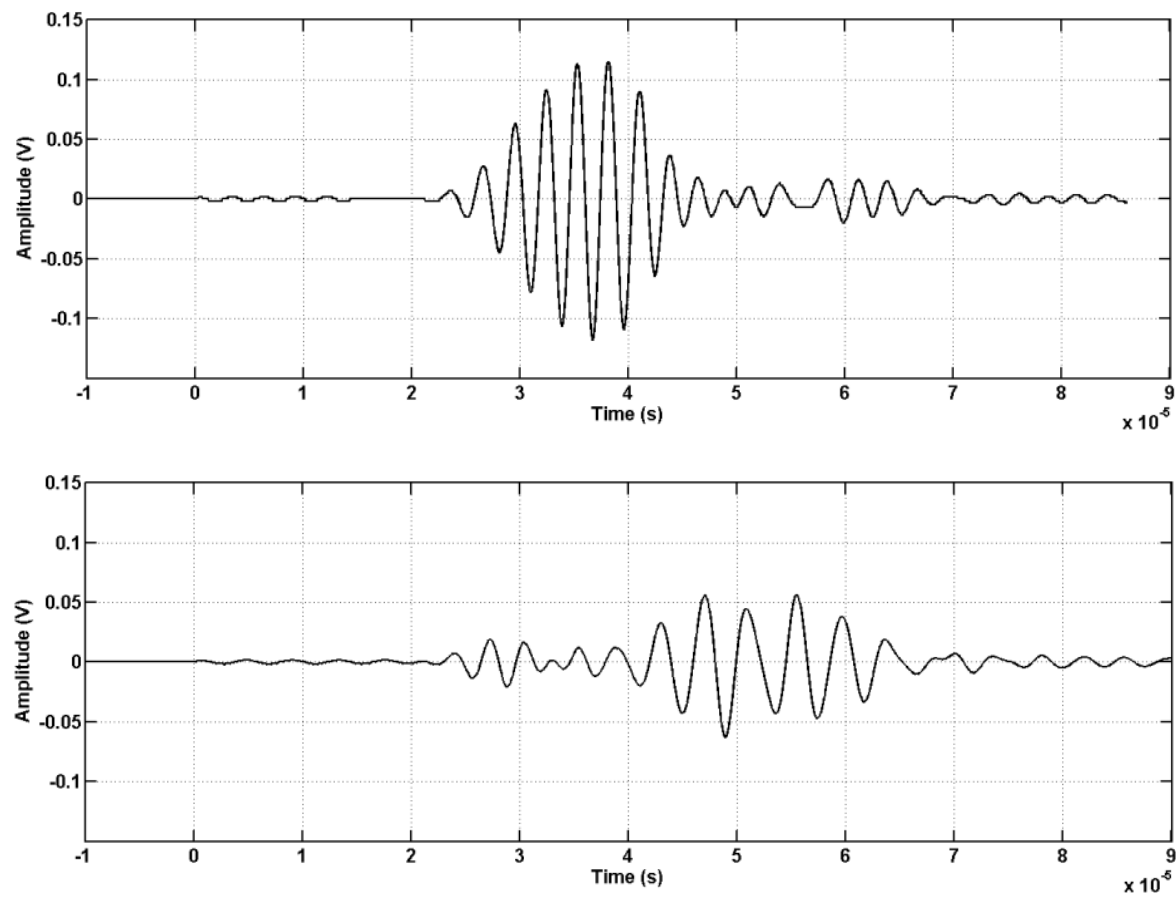

Fig. 4. Receiver signals measured at a PVC substrate with $1 \mathrm{~mm}$ thickness at two different excitation frequencies: $342 \mathrm{kHz}$ (above) and $235 \mathrm{kHz}$ (below). 
The receiver signal at $342 \mathrm{kHz}$, however, was not the only acoustic wave signal, which could be recorded in the frequency range of the experiments at the $1 \mathrm{~mm}$ PVC substrate and which was sensitive to touch effects. At 235 $\mathrm{kHz}$, another signal was observed with maximum peak-to-peak voltage amplitude of $119 \mathrm{mV}$ and a longer transit time of $28.4 \mu \mathrm{s}$ corresponding to a propagation velocity of 1761 $\mathrm{m} / \mathrm{s}$, which exhibited a touch sensitivity of $72 \%$ with the mode conversion tester (Fig. 4 below). Besides this signal, an additional signal with smaller amplitude appeared earlier in time, which corresponds to a much larger propagation velocity.

With increasing thickness of the substrates, the optimum excitation frequencies and the transmission times shifted slightly and the touch effect decreased, as exhibited in table 1.

Tab. 1: Substrates (materials and thicknesses), excitation frequencies, transit times, amplitudes, reduction by touch.

\begin{tabular}{|c|c|c|c|c|c|c|}
\hline $\begin{array}{c}\text { Substrate } \\
\text { material }\end{array}$ & $\begin{array}{l}\text { Thickness } \\
\text { (mm) }\end{array}$ & $\begin{array}{l}\text { Excitation } \\
\text { frequency } \\
(\mathrm{kHz})\end{array}$ & $\begin{array}{c}\text { Transit } \\
\text { times } \\
(\mu \mathrm{s})\end{array}$ & $\begin{array}{c}\text { Amplitude } \\
\text { receiver signal } \\
\text { without touch } \\
\qquad(\mathrm{mV})\end{array}$ & $\begin{array}{l}\text { Amplitude } \\
\text { receiver signal } \\
\text { with touch } \\
(\mathrm{mV})\end{array}$ & $\begin{array}{c}\text { Percentage } \\
\text { decrease } \\
\text { by touch }\end{array}$ \\
\hline \multirow{2}{*}{ Aluminum } & 1 & 928 & 11.6 & 751.6 & 175.4 & $77 \%$ \\
\hline & 2 & 1023 & 13.2 & 600.8 & 281.7 & $53 \%$ \\
\hline \multirow{4}{*}{ PVC } & \multirow{2}{*}{1} & 342 & 22.6 & 232.9 & 80.7 & $65 \%$ \\
\hline & & 235 & 28.4 & 119.0 & 33.9 & $72 \%$ \\
\hline & \multirow{2}{*}{2} & (394) & n.m. & 44.2 & 20.8 & $53 \%$ \\
\hline & & 224 & 33.9 & 78.7 & 25.1 & $68 \%$ \\
\hline \multirow{4}{*}{$\begin{array}{l}\text { Phenolic } \\
\text { resin }\end{array}$} & \multirow{2}{*}{1} & 389 & 17.1 & 394.8 & 245.7 & $38 \%$ \\
\hline & & 249 & 22.1 & 158.2 & 36.6 & $77 \%$ \\
\hline & \multirow{2}{*}{2} & $(439)$ & 22.0 & 78.8 & 43.3 & $45 \%$ \\
\hline & & 264 & 26.7 & 225.4 & 70.0 & $69 \%$ \\
\hline
\end{tabular}

n.m.: Time not measurable because of interfering signals.

( ): Frequency not exactly determinable because of interfering signals.

\section{Discussion of the results}

Compared with previous experiments using substrates with homogeneous thickness larger than the wavelength of the surface acoustic waves [3], the touch sensitivity turned out to be considerably larger in the present case with substrate thicknesses smaller than the wavelength. This effect has been observed with all materials investigated so far; in particular, plastic materials appeared to be touching sensitive as well.

Due to the dispersive character of Lamb waves, however, mode splitting occurred in particular at substrates with low wave velocities and correspondingly small excitation frequencies resulting from the fixed wavelength implied by the transducer pitch. This effect has been observed in particular at plastic substrates as shown in Fig. 4. The interpretation of the different modes in terms of symmetric and anti- symmetric fundamental and higher Lamb wave modes according to a dispersion diagram [6] is complicated, however, by the geometry of the samples: Since the thinned region comprises only the space between the two transducers, whereas it is surrounded by the thicker unthinned substrate, the boundary condition requirements for the calculation of dispersion relations of Lamb waves, i.e. plates with free edges, are not fulfilled in this case. Therefore, dispersion relation calculations for free plates will not be representative for the present case.

Although dispersion relation calculations for the given boundary conditions are beyond the scope of this contribution, dispersion diagrams for free plates of the same thickness may allow a tentative interpretation of the PVC results in terms of a faster symmetric mode at higher frequency and a slower anti-symmetric mode at lower frequency. Nevertheless, since the 
interaction of both modes with a touching finger may be different due to the different surface displacements in both modes, a comparison of the touch influence on both modes may provide an internal reference. In addition, a second transmission line on the thick part of the substrate will experience only a small influence from touch events and thus may be used for normalization and for temperature compensation.

There might be some doubts whether the thinned regions may withstand the forces of touch events. However, their extension may be reduced by smaller transducers and they may become stabilized by filling the hollow space with rigid foam, which, on the other hand, will not influence the propagation of surface acoustic waves because of its different acoustic impedance. In this way it seems possible to achieve a sufficient mechanical stability.

\section{Summary and outlook}

With Lamb waves propagating along thinned regions of solid substrates large sensitivities against touch effects can be obtained on different types of materials including ceramics, metals and plastics. In addition, internal references with respect to aging or temperature influences may be realizable by using several transmission lines with different thicknesses or different Lamb wave modes detectable on thin regions. Sufficient mechanical stability seems to be achievable by constructive efforts, e.g. filling the hollow space with rigid foam.

Since this touch sensing concept is widely independent of the substrate material, various objects may be made touch sensitive with this technology. Particular promising applications are e.g. touch sensitive ceramic tiles in Fig. 5 with switching properties in bathrooms or touch control panels made from metal or plastic materials at medical equipments, in kitchens or in cars.
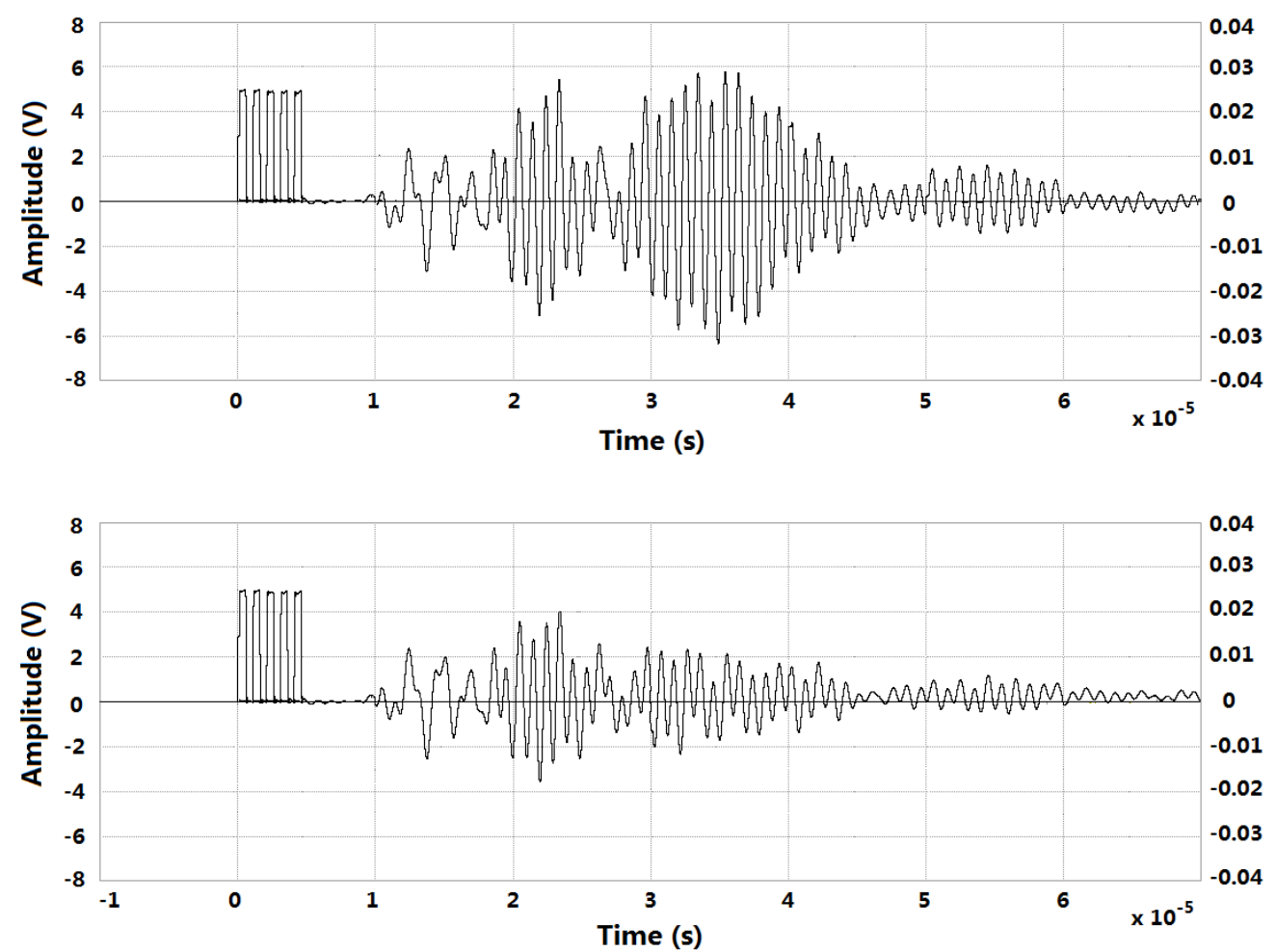

Fig. 5. Receiver signals measured at a ceramic tile with $1 \mathrm{~mm}$ thickness at excitation frequencies of $1000 \mathrm{kHz}$ without (above) and with applying human fingers (below).

\section{Acknowledgement}

The supports of Thomas Vogt with respect to the construction of the samples, of Anja Bosecker, Sabine Baier and Sabrina Tietze with respect to the measurements, of Liang Wei with respect to dispersion relation calculations, of Claudia Wolny with respect to the illustration are gratefully acknowledged. Besides, during the experiment, great help and valuable suggestions from all the colleagues from ISAT are greatly appreciated. The investigations have been funded in parts by the innovation program "Aufbruch Bayern" of the Bavarian state government. 


\section{References}

[1] R. Adler and P.J. Desmares, An Economical Touch Panel Using SAW Absorption, IEEE Transactions on Ultrasonics, Ferroelectrics and Frequency Control, Vol. UFFC-34, 195-201 (1987); doi: 10.1109/T-UFFC.1987.26932

[2] G. Lindner, Sensor and Actuators based on surface acoustic waves propagating along solide-liquid interfaces, Journal of Physics D: Applied Physics 41, 1-13 (2008); doi: 10.1088/0022-3727/41/12/123002

[3] L. Meisenbach, W. Diller, X. Zeng, M. Schmitt, C. Hamperl and G. Lindner, Berührungssensitive Fliesen auf Basis von akustischen Oberflächenwellen für den Sanitärbereich, in Tagungsband 4. Deutscher Aal-Kongress, Berlin 2011, VDE Verlang Berlin Offenbach, 2011 (Poster 1.7)

[4] I.A. Viktorov, Rayleigh and Lamb waves, Plenum Press: New York, 1967

[5] J.L. Rose, Ultrasonic Waves in Solid Media, Cambridge University Press: Cambridge, 1999

[6] H. Faustmann, M. Münch, G. Lindner, M. Schmitt and M. Springer, Measurement of the properties of liquids based on the dispersion of Lamb waves in an acoustic waveguide, Physics Procedia 3, 959-964 (2010); doi: 10.016/j.phpro.2010.01.123 